EPJ Web of Conferences 92,02121 (2015)

DOI: $10.1051 /$ epjconf/ 20159202121

(C) Owned by the authors, published by EDP Sciences, 2015

\title{
Analysis of radiometric signal in sedimentating suspension flow in open channel
}

\author{
Marcin Zych ${ }^{1, a}$, Robert Hanus ${ }^{2}$, Leszek Petryka ${ }^{1}$, Dariusz Świsulski ${ }^{3}$, Marek Doktor ${ }^{1}$ and Wojciech Mastej $^{1}$ \\ ${ }^{I}$ AGH University of Science and Technology, Krakow 30-059, Poland \\ ${ }^{2}$ Rzeszow University of Technology, Rzeszow 35-959, Poland \\ ${ }^{3}$ Gdansk University of Technology, Gdansk 80-233, Poland
}

\begin{abstract}
The article discusses issues related to the estimation of the sedimentating solid particles average flow velocity in an open channel using radiometric methods. Due to the composition of the compound, which formed water and diatomite, received data have a very weak signal to noise ratio. In the process analysis the known determining of the solid phase transportation time delay the classical cross-correlation function is the most reliable method. The use of advanced frequency analysis based on mutual spectral density function and wavelet transform of recorded signals allows a reduction of the noise contribution.
\end{abstract}

\section{Introduction}

Movement of mineral particles may vary in a broad range and has crucial influence on the new geological layers creation [1-3].

The ability to simulate a slurry current in a laboratory open channel enables a study of the geological formations mechanisms, including geochemical and geological processes of hydrocarbon traps. From other site examination of flows in rocks on a microscale may help among other in extraction of oil and natural gas [4]. In addition, some industrial processes, such as handlers of water treatment are also often done in open channels.

The authors focus on using radiometric equipment in open channel measurement $[2,3,5,6]$, which may be a complementary method to other investigations procedures [7-10].

\section{Radiometric absorption method}

Figure 1 shows a measurement in the channel with installed absorption set, comprising two sealed radioactive sources and two scintillation probes. As a source of the gamma radiation, two sealed ${ }^{137} \mathrm{Cs}$ isotopes with activities of $100 \mathrm{mCi}$, placed in collimators (2) spanned of $L=90 \mathrm{~mm}$ were applied. Exactly at the opposite trough's side a couple of probes with $\mathrm{NaI}(\mathrm{Tl})$ scintillation detectors were installed in the collimators (3). The photon beam (4), which is shaped by the source collimator, passes through the flowing slurry, and some photons are absorbed depending on density and content of the flow. The rest of the photons reach the probes, and produce counts rates $I_{x}(t)$ and $I_{y}(t)$. The resulting signals are stochastic and because of the geometry of the measurement and a small density of diatomite they have a low signal - noise ratio.

\section{Laboratory stand}

The general view of the research installation is presented in figure 2. It was built in the Sedimentological Laboratory of the Faculty of Geology, Geophysics and Environment Protection of the AGH University of Science and Technology in Krakow. The installation schema is presented in figure 3 . It is comprised of an open channel $W=500 \mathrm{~mm}$ width, $D=200 \mathrm{~mm}$ depth and $H=400 \mathrm{~mm}$ high (1) with glass walls used for examination of slurry sedimentation and transport. The flow from the feed tank (2) through the trough goes gravitationally to the overflow tank (3) and is returned by a sludge feed pump (4) via a pipe (5). The prepared beforehand a sedimentation compound may be supplied to the installation through the overflow tank (3) or a rock spall conveyor (8). The slurry rate may be control by adjustment of the stream height (6) and throttle valve (7) position. Moreover, the channel inclination angle may be set by a screw support adjustment. The gamma absorption set was placed on a special measuring trolley (9), while the ultrasound meter (10) was secured directly at the trough wall. The trolley (9) enables not only the absorption set to be installed, but also the installation of other equipment e.g. a depth gauge or a camera. The applied ultrasound meter Controlotron UNIFLOW 990E allows the liquid velocity measurements to be done with an uncertainty below $1 \%$.

\footnotetext{
a Corresponding author: zych@geol.agh.edu.pl
} 


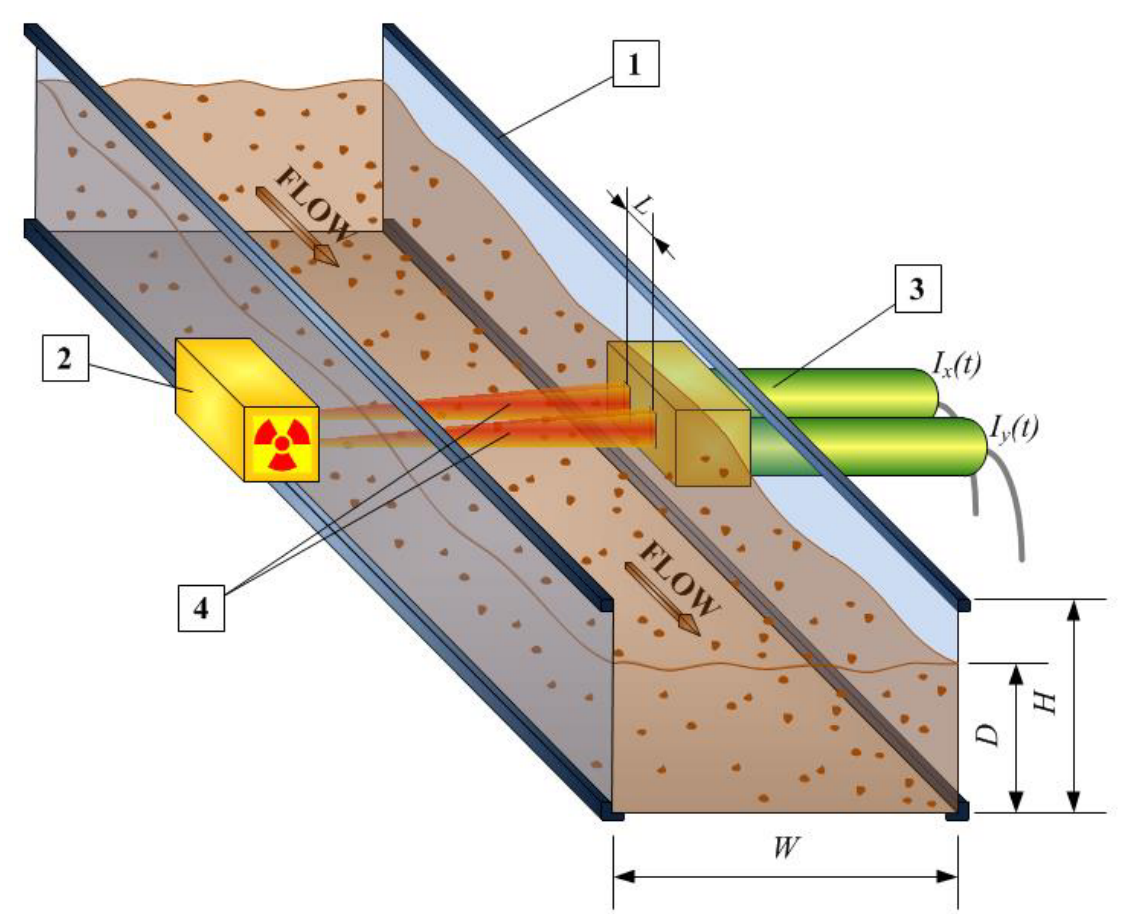

Figure 1. Idea of two-phase flow measurement in an open channel by $\gamma$-ray absorption method: 1 - open channel with the flowing mixture of 2 - collimator with two gamma-ray sources ${ }^{137} \mathrm{Cs}, 3$ - a set of two scintillation probes $\mathrm{NaI}(\mathrm{Tl})$ with collimator, $4-\gamma$-ray beam.

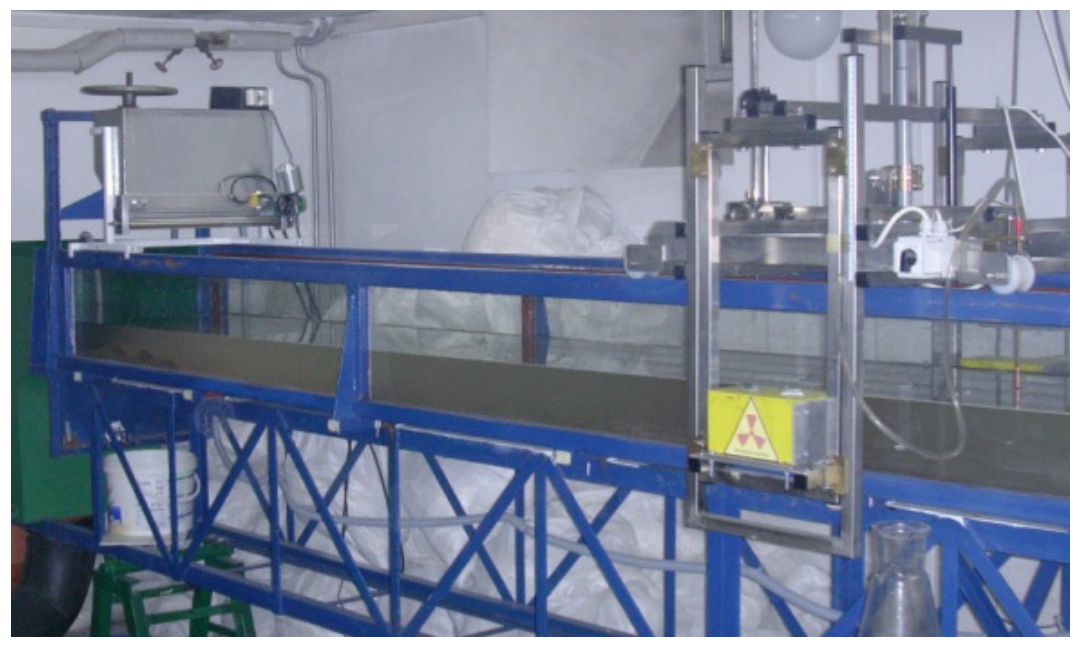

Figure 2. General view of the research installation with the radioisotope absorption set installed on the measuring trolley.

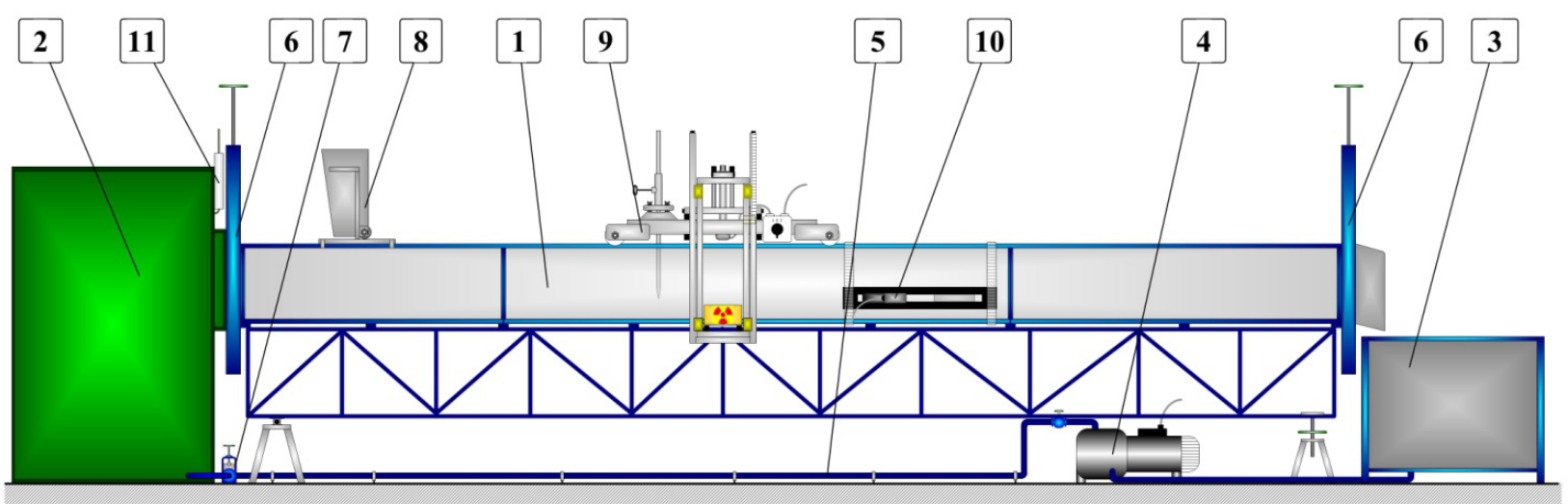

Figure 3. Schema of installation: (1) open channel, (2) the feed tank, (3) the overflow tank, (4) a sludge feed pump, (5) connecting pipe, (6) the sluicegate, (7) throttle valve, (8) a rock spall conveyor, (9) an special measuring trolley, (10) the ultrasound meter, (11) a brief water-level gauge. 
In addition, the installation was equipped with a brief water-level gauge (11), used like the ultrasound meter for evaluation of the averaged water velocity, but with lower accuracy.

\section{Signal analysis and exemplary results}

The voltage pulses from probes are recorded with $\Delta t=1 \mathrm{~ms}$ sampling rate and single record contain $N=300000$ samples. Figure 4 show $I_{x}(t)$ and $I_{y}(t)$ signals obtained in run GGO0010.

a)

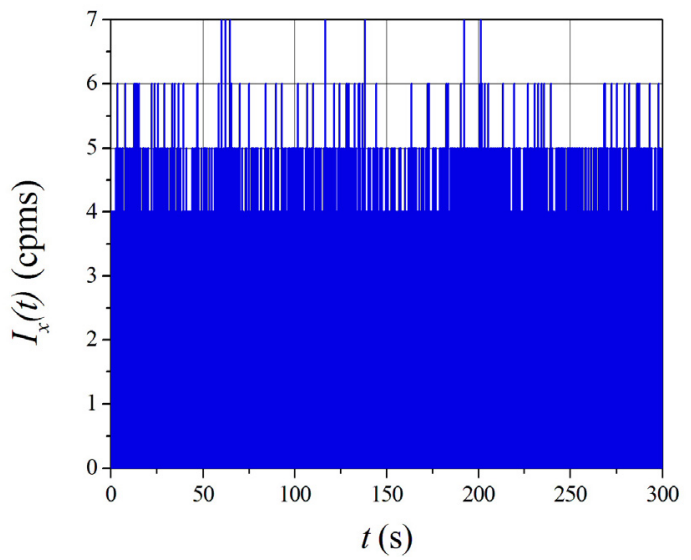

b)

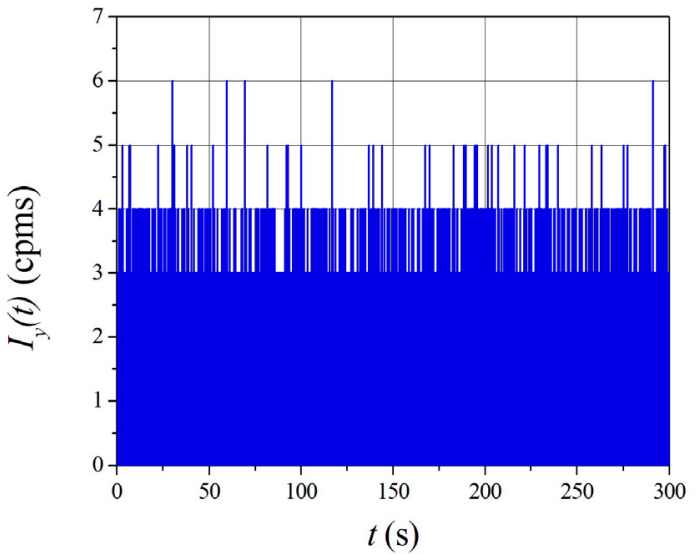

Figure 4. Exemplary signals received from the scintillation probes for run GGO0010.

The determination of the average solid particles transportation time delay $\tau_{0}$ is necessary to specify the velocity of solid phase between detectors. Due to low signal-to-noise ratio (SNR), the classical cross-correlation function (CCF) has to be applied [11-14].

The CCF is defined as follows:

$$
R_{x y}(\tau)=\lim _{T \rightarrow \infty} \frac{1}{T} \int_{0}^{T} x(t) \cdot y(t+\tau) d t
$$

where: $T$ - averaging time, $\tau$ - time delay.

Determination of transportation time delay $\tau_{0}$ is based on finding the position of main maximum of the CCF:

$$
\tau_{0}=\arg \left\{\max R_{x y}(\tau)\right\}=\arg \left\{R_{x y}\left(\tau_{0}\right)\right\} .
$$

However, the resulting distribution for the GGO0010 run, has a significant share of noise, so it is hard to precisely determine the transportation time delay. For the signal-to-noise ratio improvement the following methods were applied:

- digital filtration based on signal analysis in the frequency domain,

- noise reduction by the discrete wavelet transform (DWT).

In the first case proper reduction of boundary frequencies improve the useful part of the cross-spectral density function (CSDF) [12-14]:

$$
G_{x y}(f)=2 \int_{-\infty}^{\infty} R_{x y}(\tau) \cdot e^{-j 2 \pi \cdot f \cdot \tau} d \tau
$$

Constructed in this way a bandpass filter rejects undesirable low and high frequency components. The obtained correlogram is shown in figure $5 \mathrm{a}$.

a)

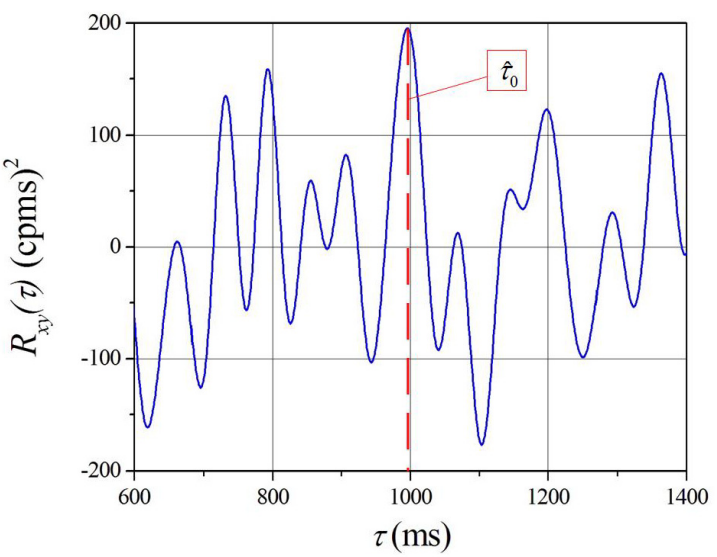

b)

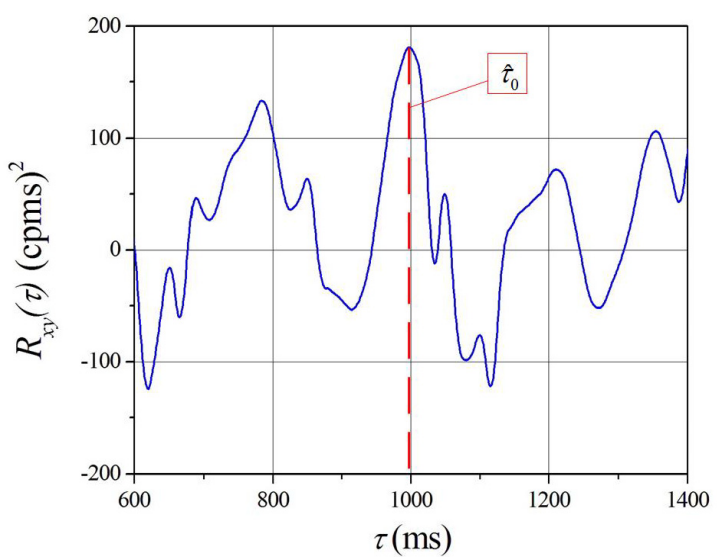

Figure 5. Cross-correlation function after: (a) the application of bandpass filtering, (b) denoising using wavelet transform. Dashed line indicates the time delay.

The second method uses the discrete wavelet transform (DWT), which found practical application for the first time in the analysis of seismic data by Morlet [15] and is performed in the time domain. Currently DWT is widely used in the signal processing for noise reduction, compression, etc., not only in geophysics but also medical diagnostics and other technical fields [15$18]$. 
By means of DWT the $I_{x}(t)$ signal can be represented as [15-17]:

$$
I_{x}(t)=\sum_{m=0}^{M-1} \sum_{n=0}^{N-1} d_{m, n} \cdot g_{m, n}(t)
$$

wherein $d_{m, n}$ are the coefficients calculated in the equation:

$$
d_{m, n}=\int I_{x}(t) \cdot \gamma_{m, n}^{*}(t) d t
$$

In turn, $g_{m, n}(t)$ are base functions arising from scaling and translation of the mother wavelet $\chi(t)$ :

$$
g_{m, n}(t)=2^{-m / 2} \cdot \gamma\left(2^{-m} t-n\right) .
$$

$\gamma_{m, n}(t)$ function is a dual to $g_{m, n}(t)$ one. Most of the currently used wavelets are orthogonal functions. However, a family of wavelets exist, which are not orthogonal, but biorthogonal (bior) one. Such functions are only orthogonal to each other in pairs, and in many cases significantly better bulge the characteristics of the signal. An important application of the DWT is the level rejection details of analyzed information. In this case the biorthogonal 3.1 wavelet was applied, where details were rejected at the level of 6 . The effect of this processing of the CCF is shown in figure $5 \mathrm{~b}$.

Uncertainties of the average transportation time delay determination based on the standard deviation $\sigma$ of the Gaussian distribution fitted to the CCF peak, then:

$$
u_{A}\left(t_{0}\right)=\frac{\sigma}{\sqrt{z}}
$$

where: $z$ - number of points selected for fitting procedure

After determining the time $\hat{\tau}_{0}$ and knowing the distance $L$ between the probes it is possible to calculate the average flow velocity of solid particles $[2,3,5]$ :

$$
v_{S}=\frac{L}{t_{0}}
$$

Since the average velocity of this phase is determined in an indirect way, so to estimate the result accuracy, the propagation of uncertainty was used [19]:

$$
u_{C}\left(v_{S}\right)=\sqrt{\left[\frac{\partial v_{S}}{\partial L} \cdot u_{B}(L)\right]^{2}+\left[\frac{\partial v_{S}}{\partial t_{0}} \cdot u_{A}\left(t_{0}\right)\right]^{2}}
$$

where: $u_{B}(L)$ - the standard uncertainty of the distance of the deployed detectors measurement (for this set equal to $0.1 \mathrm{~mm}), u_{A}\left(\hat{t}_{0}\right)$ - the standard uncertainty of the average transportation delay determination.

The $A$ and $B$ indexes stand for respective uncertainties of type A and B.

In table 1 the results of estimation of transportation time delay and average velocity of solids obtained for run GGO0010 are listed. In addition $v_{W}$ water velocity, measured by the ultrasound flow meter is added.
Table 1. Results of time delay and velocity estimation using $\mathrm{CCF}$ and two methods of noise reduction for run GGO0010

\begin{tabular}{|c|c|c|c|c|c|}
\hline $\begin{array}{c}\text { Denoising } \\
\text { method }\end{array}$ & $\begin{array}{c}\hat{\tau}_{0} \\
\mathrm{~ms}\end{array}$ & $\begin{array}{c}u_{A}\left(\hat{\tau}_{0}\right) \\
\mathrm{ms}\end{array}$ & $\begin{array}{c}v_{S} \\
\mathrm{~ms}\end{array}$ & $\begin{array}{c}u_{C}\left(v_{S}\right) \\
\mathrm{ms}\end{array}$ & $\begin{array}{c}v_{W} \\
\mathrm{~ms}\end{array}$ \\
\hline$|\mathrm{CSDF}|$ & 994.4 & 2.0 & 0.0905 & 0.0002 & \multirow{2}{*}{0.134} \\
\cline { 1 - 5 } DWT & 994.6 & 2.2 & 0.0905 & 0.0002 & \\
\hline
\end{tabular}

\section{Conclusions}

The presented measuring system based on the absorption of gamma radiation allows determination of the average flow velocity of sedimenting solid particles. Improvement of transport time delay estimation for signals with low signal to noise ratio is possible due to the use of the cross-correlation function and filtration performed in the frequency domain by analyzing the cross spectral density function, or in the time domain based on the discrete wavelet transform for recorded signals. Both methods give similar values of $\tau_{0}$ (figure 5) and average solid phase velocity, with the uncertainty of several per mille (table 1). Such uncertainties obtained in the exemplary measurement GGO0010 not exceed $0.5 \%$ of average velocity of solid particles, which proves the precision of the measurement. Very similar results were obtained in the other laboratory measurements [2].

In addition, the use of an ultrasonic meter, allow determination of average water flow velocity and flow rate in the channel, which is required for investigation of the phases interactions and verification of mathematical models of solid particles transportation phenomena.

Moreover gamma-ray absorption as noninvasive method can be used in investigations of flows in open channels or pipelines. Constrain in those proposal arise mostly from rigorous safety regulations reducing the radiation hazard during application of radioactive isotopes.

\section{References}

1. G. Einsle, Sedimentary basins, evolution, facies, and sediment budget (Springer, Berlin, 2000)

2. M. Zych, et al., Flow Meas. Instrum. 35, 11-15 (2014)

3. M. Zych, et al, Prz. Elektrotech. 88 (10a), 99-100 (2012)

4. P. Krakowska, P. Madejski, J. Jarzyna, 75th EAGE conference \& exhibition incorporating SPE EUROPEC 2013, 1-5, (2013)

5. L. Petryka, et al, Prz. Elektrotech. 86 (5), 24-29 (2010)

6. A.B. Tuğrul, N. Altınsoy, Flow Meas. Instrum. 12, 341-344 (2002)

7. W. Błotnicki, J. Wiora, S. Waluś, Prz. Elektrotech. 89 (3a), 293-297 (2013)

8. V. Weitbrecht, G. Kühn, G.H. Jirka, Flow Meas. Instrum. 13, 237-245 (2002) 
9. M.C. Lee, J.M. Leu, H.C. Chan, W.C. Huang, Flow Meas. Instrum. 21, 150-154 (2010)

10. A. Michalski, W. Piotrowski, IEEE Trans. Instrum. Meas. 51 (4), 833-839 (2002)

11. L. Petryka, J. Oszajec, Nucl. Geophys. 7 (2), 323333 (1993)

12. J.S. Bendat, A.G. Piersol, Random data - analysis and measurement procedures (John Wiley, New York, 2010)

13. M.S. Beck, A. Pląskowski, Cross-correlation flowmeters (Bristol, Adam Hilger, 1987)

14. L. Tan, J. Jiang, Digital signal processing. Fundamentals and applications (Oxford, Elsevier, 2013)
15. P. Augustyniak, Wavelet transforms in electrodiagnostic applications (Wydawnictwa AGH, Krakow 2003)

16. J.-L. Starck, F. Murtagh, J. M. Fadilli, Sparse image and signal processing. Wavelets, curvelets, morphological diversity (New York, Cambridge University Press, 2010)

17. S. Degaleesan, M.P. Dudukovic, Y. Pan, Flow Meas. Instrum. 13, 31-43 (2002)

18. K. Wawrzyniak, Acta Geophys. 58 (1), 49-82 (2010)

19. Guide to the expression of uncertainly in measurement (International Organization for Standardization 1995) 